\title{
Application of targeted next-generation sequencing in SNHL patients
}

\author{
Dobriyanova V. \\ Ear, Nose, Throat Diseases Department, Faculty of Medicine at Sofia Medical University
}

\begin{abstract}
Sensorineural hearing loss, SNHL, is a complex disease impacted by the interaction of a multitude of endogenous and exogenous factors. Genetic and age-related changes predetermine the state of hearing. Genetic factors and factors affecting development play a key role in the occurrence of SNHL. The goal of this study is to research functioning of the hearing organ in patients with a family history of reduced hearing, to analyse the causes of deafness, to provide an identification strategy of genetic causes and to refer the patient to the most effective treatment.

The study included 192 patients: 178 of them with sensorineural hearing loss and 14 subjects with normal hearing, relatives of the study patients with impaired hearing, of who biological material was also taken to look for mutations associated with hearing. Screening of the patients participating in the study started with searching for mutations in Cx26 (GJB2), Cx30 (GJB6) and Cx31 (GJB3). At the next stage, five families were chosen from the patients without a mutation in the screened genes. They underwent targeted nextgeneration sequencing in search of rare genes causing SNHL. Clinical, audiological and genetic methods were used in conducting the study.

In $31.77 \%$ of the cases the reason for deafness was identified (a genetic reason was found) of which $29.69 \%$ cases $(n=57)$ were mutations in Cx26 (in a homozygous state), corresponding with data published in world's literature on mutations in Cx26 of between 18 to $40 \%$ in cases of patients with SNHL. In the remaining $2.08 \%$ of the patients, rare mutations responsible for the deafness were identified.
\end{abstract}

\section{Introduction}

Nowadays, there are increased opportunities for diagnostic assessment and treatment of SNHL. In most of the cases, hearing loss has a multifactorial genesis, the causes being both genetic and resulting from environmental factors. A gene mutation can lead to hearing loss. In these cases hearing loss is a monogenetic disorder with autosomal dominant, autosomal recessive, $\mathrm{X}$-linked or mitochondrial inheritance.

Hearing loss can be classified as genetic or non-genetic, depending on the presence of coding mutations; depending on the time of occurrence it can be prelingual or postlingual; and syndromic (a combination of hearing loss and one or more other specific anomalies) or non-syndromic.

Dozens of genetic loci have been identified as the reason for syndromic and non-syndromic hearing loss with various models of inheritance (autosomal dominant, autosomal recessive, X-linked, mitochondrial). The prelingual sensorineural hearing loss is estimated at 1 in 500 children in developed countries as in $80 \%$ of the cases hearing loss is genetic. About $80 \%$ of the affected have non-syndromic hearing loss while the remaining $20 \%$ syndromic. In $80 \%$ of the cases the non-syndromic hearing loss is inherited in a recessive pattern, in $19 \%$ the inheritance is autosomal dominant and in less than $1 \%$ the modes of inheritance are mitochondrial, miRNA and X- linked [1].

The main goal of our study is to provide an identification strategy of the genetic causes and to refer 
the patient to the most effective treatment. For the purposes of implementing that goal, a genetic screening algorithm was developed (Figure 1).

During the screening of the study participants, a mutation in GJB2, the gene encoding protein connexin 26 was identified, as the frequency was $43.75 \%$. In $29.69 \%$ of the cases, it involved mutant alleles in a homozygous state, while in $14.06 \%$ of the cases mutations were in a heterozygous state. Mutations in the GJB2 gene are the most frequent cause of monogenic hearing loss, responsible for about half of all the cases of autosomal recessive prelingual hearing loss. It is inherited in an autosomal recessive pattern, which means that two copies of the GJB2 gene have to be changed in each cell to cause a hearing impairment. Mutations in this gene alter the gap junctions, which causes disruption in the levels of potassium ions in the inner ear.

The screening continued with searching for mutations in the genes GJB6 and GJB3, encoding proteins connexin 30 and connexin 31 respectively. Among the 192 study patients, mutation of the gene encoding Cx30 was not found, which is not unusual in view of its low frequency in the population $0.65 \%$ [2]. Mutations in $\mathrm{Cx} 31$ were not identified either.

As a next step in diagnosing hereditary hearing loss, we introduced the application of targeted next-generation sequencing [Figure 1]. We selected families where several members of different generations were affected by SNHL and our aim was to identify rare mutations in genes encoding hearing.

\section{Genetic screening algorithm}

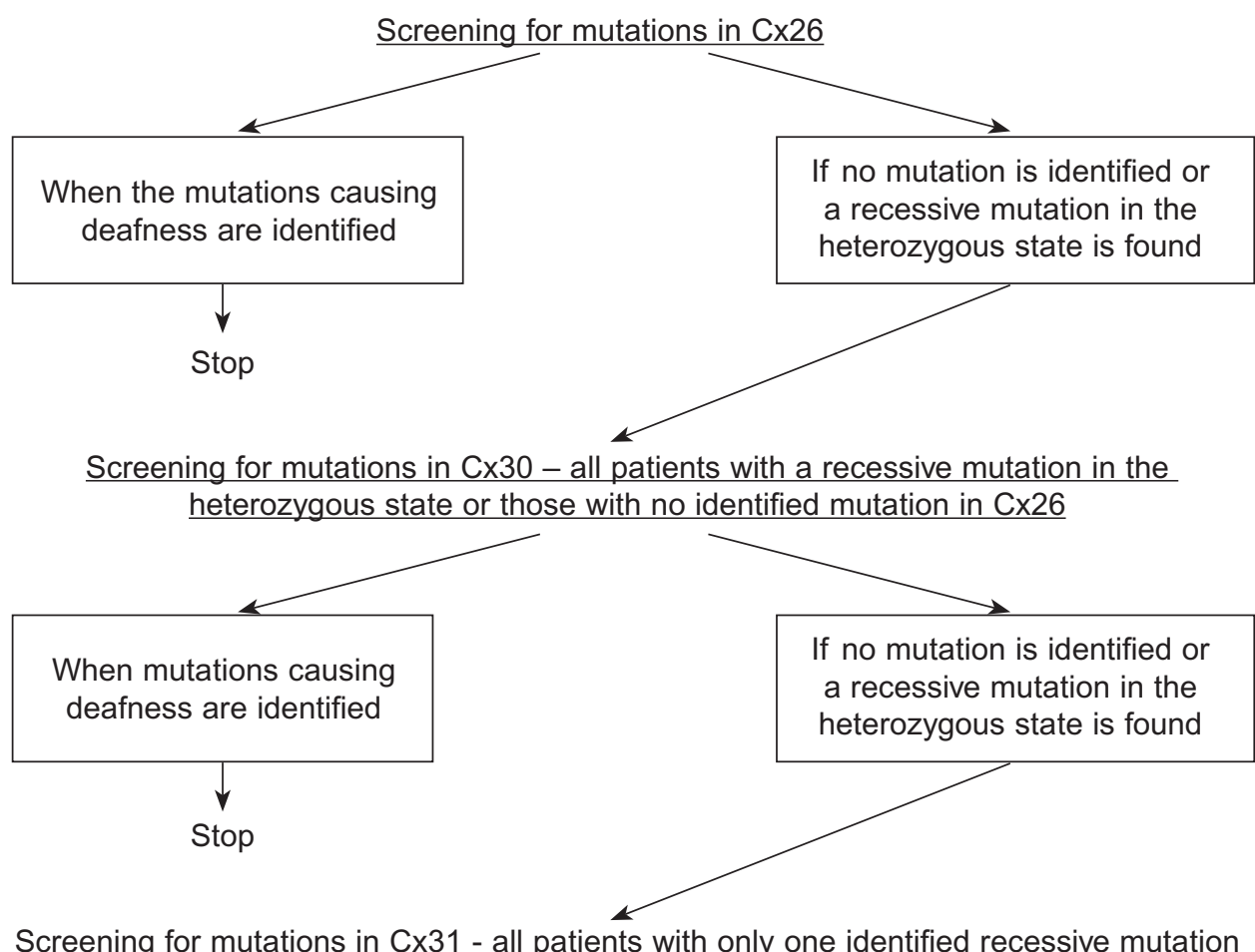

in the heterozygous state in $\mathrm{C} \times 26$ or $\mathrm{Cx} 30$ and all patients with no identified mutation

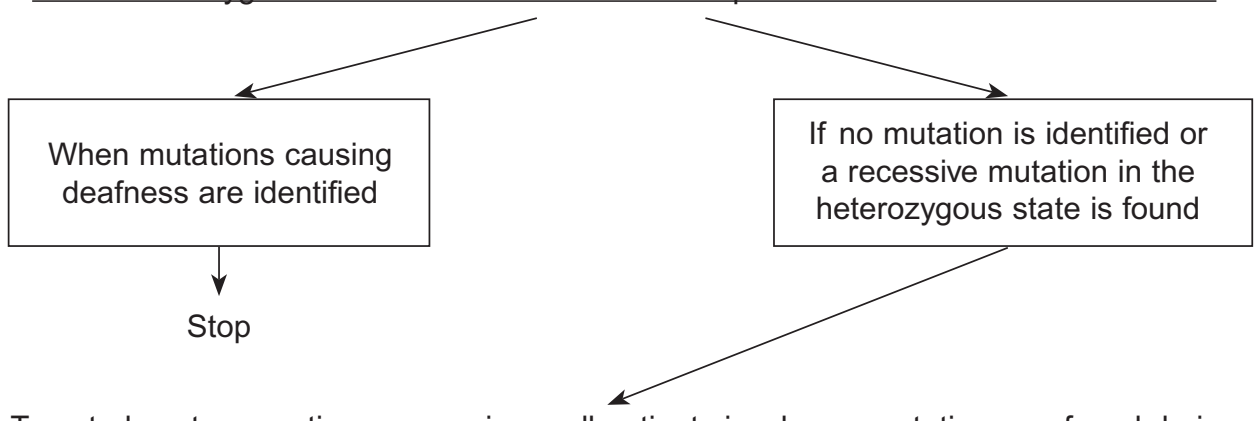

Targeted next-generation sequencing - all patients in who no mutation was found during the screening for common mutations (in $\mathrm{C} \times 26, \mathrm{C} \times 30$ and $\mathrm{C} \times 31$ ), causing deafness.

Figure 1. Genetic screening algorithm. 
Due to the substantial genetic heterogeneity of deafness, the clinical application of genetic information is still leading to a number of difficulties. It is known that more than 80 genes are related to the non-syndromic hereditary hearing loss. Progress in targeted exome sequencing of selected genes, using massively parallel DNA sequencing allows the successful identification of relatively rare gene mutations [3].

\section{Materials and methods}

The study included 192 patients: 178 of them with sensorineural hearing loss and 14 subjects with normal hearing, relatives of the study patients with impaired hearing, of who biological material was also taken to look for mutations associated with hearing.

According to anamnestic data in $54.17 \%(\mathrm{n}=104)$ of the cases hearing loss occurred prelingually, before development of speech, in $38.54 \%(n=74)$ of the cases postlingually, after acquiring speech and $7.29 \%(\mathrm{n}=14)$ of the cases had socially adequate hearing ability.

The diagnosis was established through pure-tone threshold audiometry of all participants over 6 years of age. Play audiometry was performed in patients between the age of 3 and 6 and for children younger than 3, BAEPs were used. Otoscopy, tympanometry and reflexometry were performed in all participants.

When selecting the patients the following criteria were met:

- Patients were studied regardless of their age, gender and ethnicity;

- Enrolled patients had bilateral sensorineural hearing loss, irrespective of the degree of hearing loss;

- The study also included normally hearing subjects with a family history of SNHL;

- All study participants have a normal ENT status and respectively normal middle ear pressure;

- Patients with syndromic sensorineural hearing loss were not studied;

- In studied patients, hearing impairments caused by exogenous factors such as craniocerebral trauma, noise trauma, exposure to loud noise were excluded.

The mean age of the study participants was 20.4 years, the youngest being two-months old and the oldest one - 70 years old.

From the study patients $73.44 \%(n=141)$ identi- fied themselves as Bulgarians, 16.15\% $(\mathrm{n}=31)$ as Roma, 5.73\% ( $\mathrm{n}=11)$ as Turks and 4.69\% $(\mathrm{n}=9)$ came from mixed marriages.

According to the Bulgarian standards 55.73\% ( $\mathrm{n}=$ $107)$ of the study patients had deafness, $18.75 \%$ $(n=36)$ of them were with severe deafness (hearing loss between 60 and $90 \mathrm{~dB}), 18.3 \%(\mathrm{n}=35)$ with moderate (hearing loss from 30 to $60 \mathrm{~dB}$ ), and $7.9 \%(n=14)$ of the subjects had normal hearing.

\section{Methods}

For the purposes of the genetic testing DNA was isolated with CHEMAGEN $\AA$ magnetic separation station. The aim of the isolation was to receive high molecular weight DNA with minimal impurities such as protein, RNA and glycoproteins. A new trend in the methods for isolation of high molecular weight DNA is through automated magnetic separation, which was used in this study.

The quality of the received high molecular weight DNA was defined through agarose gel electrophoresis using $0.8 \%$ agarose gel and 1xTBE (tris, borate, EDTA buffer). Concentration of the isolated DNA was estimated with the spectrophotometer NanoDrop ${ }^{\mathrm{TM}}$.

To perform the targeted next-generation sequencing we used TruSight One Kit for the preparation and the platform MiSeq, Illumina for the sequencing. Preparation of the libraries was done according to the manufacturer's requirements. [Figure 2]

- Tagmentation of the genomic DNA

- Purification of the tagmented DNA

- Amplification and indexing of the tagmented DNA

- Purification of the amplified DNA

- Hybridization with targeted probes

- Enrichment with hybridized probes

- Second hybridization

- Enrichment with hybridized probes

- Purification of the library

- Amplification of the enriched library

- Purification of the amplified library

- Check of the library

Figure 2. Main steps in preparation of libraries 


\section{Bioinformatics methods}

The obtained data was processed with $\operatorname{VarSeq}{ }^{\circledR}$ (Golden Helix, Inc., Bozeman, MT, www.goldenhelix.com), a software package, and the analysis included:

- Alignment and mapping of reads to the human reference genome HG19;

- Removal of duplicate reads, indel realignment and base quality score recalibration (BQSR);

- Identification of human reference genome variants;

- Annotation of public databases on population frequencies, position in the corresponding protein and mRNA, pathogenicity predictions, evolutionary conservatism, etc;

- Prioritizing the identified variants through filtering.

The functional impact of the candidate mutations was assessed in silico, using prediction programmes to determine the possible phenotypic impact on the variant. For predicting the effect of missense mutations, the following tools were used: SIFT http://sift.jcvi.org/ , Polyphen-2 http://genetics.bwh.harvard.edu/pph2/, MutationTaster http:// www.mutationtaster.org/, Meta-SNP http://snps. biofold.org/meta-snp/, MutationAssessor (mutationassessor.org), Provean (provean.jcvi.org), LRT (Chun and Fay, 2009), VEST 3 (Carter H. et al, 2013), MetaSVM; for variants potentially leading to changes in the splicing: Automated Splice Site
And Exon Definition Analyses http://splice.uwo. ca/, NetGene2 http://www.cbs.dtu.dk/services/NetGene2/, Human Splicing Finder http://www.umd. be/HSF3/HSF.html .

\section{Results}

From the 192 study patients, in $31.77 \%(n=61)$ a mutation causing hearing loss was identified while in $68.23 \%(\mathrm{n}=131)$ of the cases the reason for hearing loss remained unclear. After performing genetic tests in $43.75 \%(n=84)$ of the cases, a mutation in GJB2 was identified, the gene encoding protein connexin 26, as this percentage included both heterozygous and homozygous mutations. In $2.08 \%$ $(n=4)$ of the cases mutations causing hearing loss were found in genes other than connexin genes. In $54.17 \%(\mathrm{n}=104)$ no mutations involved in hearing were identified. [Figure 3]

In $29.69 \%(n=57)$ of the tested patients, it was found that the hearing loss is caused by a homozygous mutation in the gene encoding protein $\mathrm{Cx} 26$. In $14.06 \%(n=27)$ of the study group, mutations were identified in the gene encoding protein Cx26 in a heterozygous state. In those patients, the reason for the hearing loss remained undefined. In $2.08 \%(n=4)$ of the cases mutations causing hearing loss were found in genes other than GJB2 gene. In $54.17 \%(n=104)$ of the tested the reason for hearing loss was not identified. [Figure 4] In $57.89 \%(n=110)$ of the cases the hearing loss

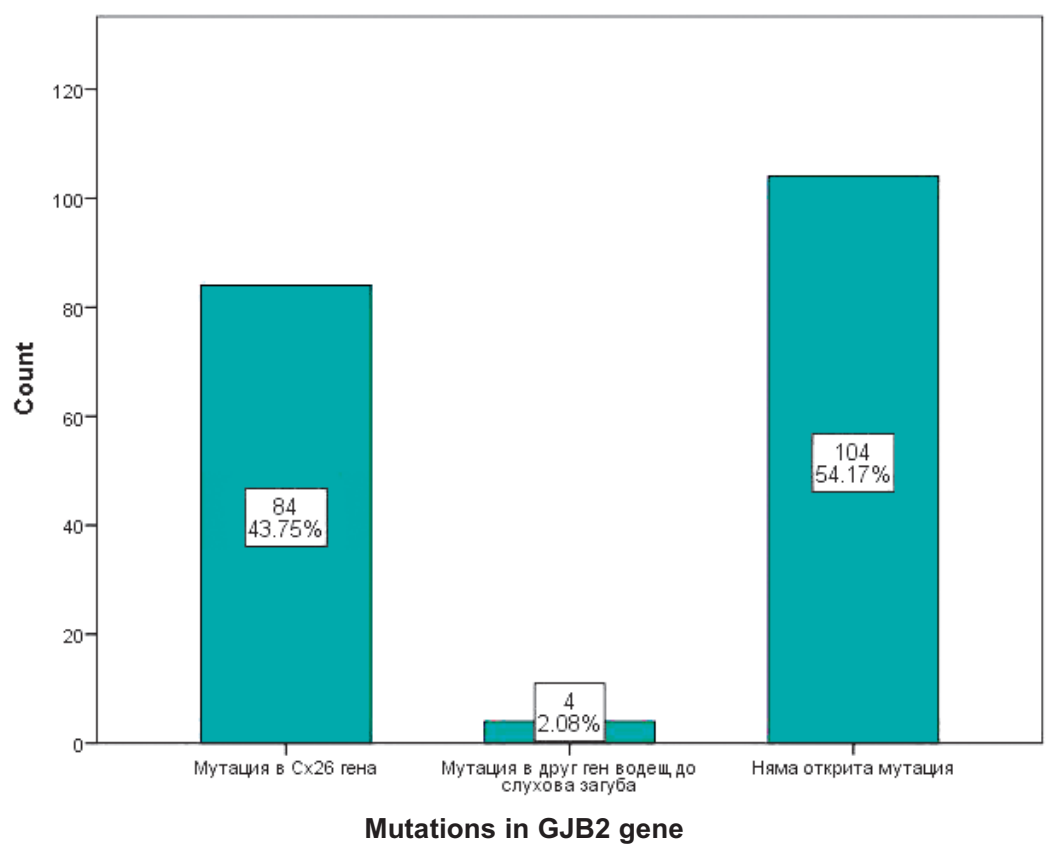

Figure 3: Types of genetic mutations in the study. 


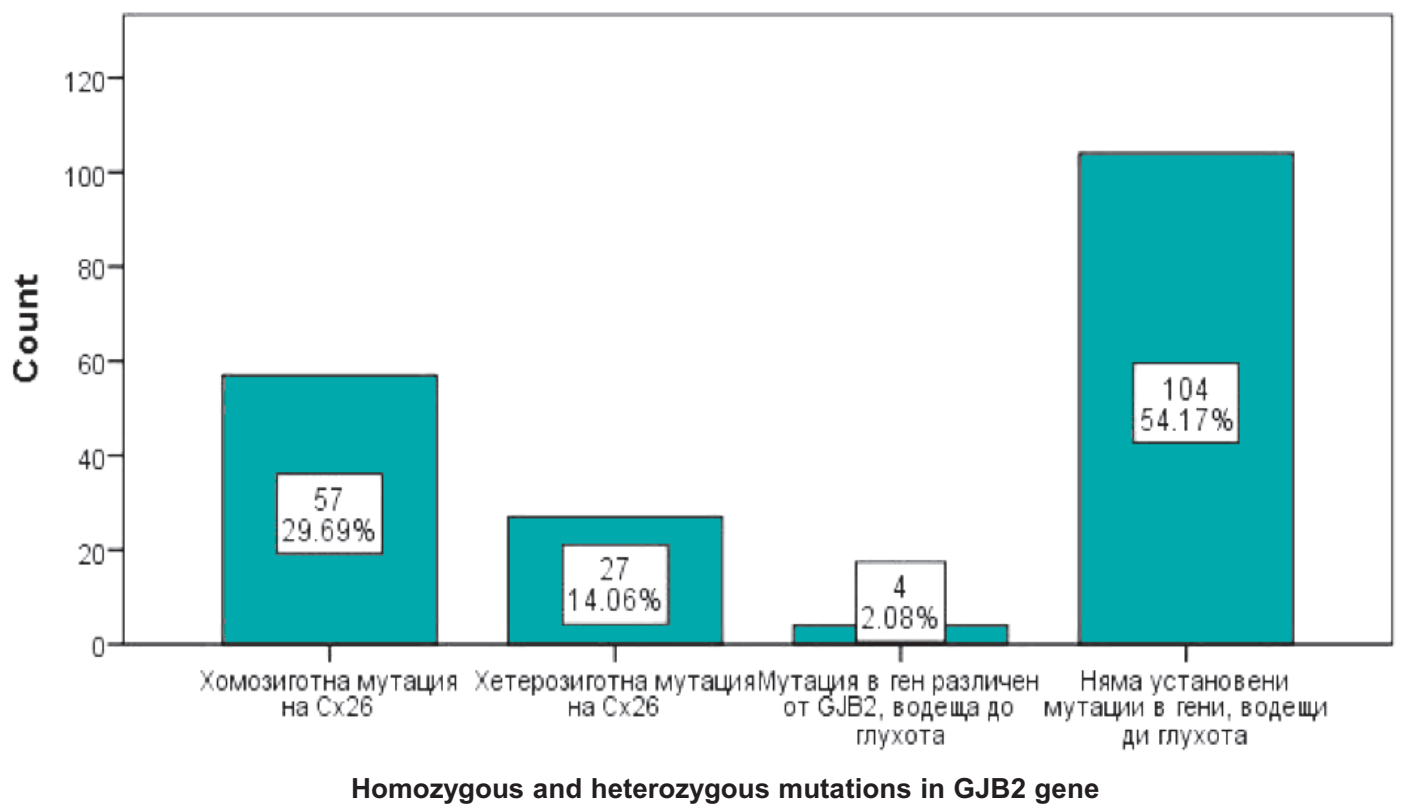

Figure 4: Types of genetic mutations in the study.

had no progression; patients with prelingual hearing loss belonged to that group. In the remaining $42.11 \%(n=80)$ of the cases hearing deteriorated over time. When comparing progression of hearing loss with regard to the impairment onset, it can be seen that the lack of progress in the hearing impairment in cases of prelingual deafness, i.e. $50.57 \%$ (n $=89$ ), dominated over the presence of progression found in $7.39 \%$ of the cases. Progression in hearing loss dominated in the postlingual impairment - in $36.93 \%(n=65)$ over the lack of progression - in $5.11 \%$ of the cases.
A comparison was made of the presence of a genetic mutation in patients of Bulgarian, Turkish, Roma and mixed ethnic origin and the results did not show statistical significance $-\mathrm{p}=0.217$. Mutations in genes causing a hearing impairment were distributed regardless of the ethnic origin. [Figure 5]

Statistical significance was found $(\mathrm{p}<0.05)$ between the presence of mutations in the genes responsible for hearing and the onset of the hearing impairment. Comparing the group of patients with hearing loss caused by a mutation with the group

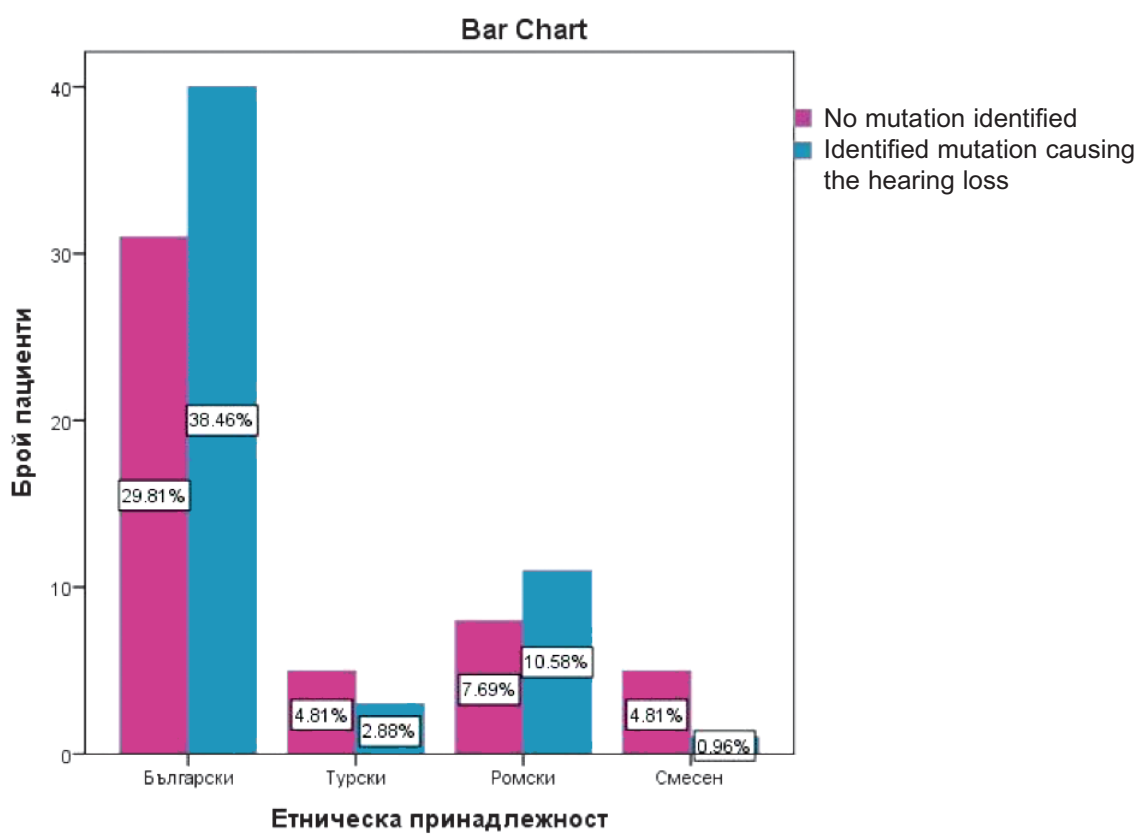

Figure 5. Genetic mutations based on ethnicity. 
of patients without identified mutations in genes involved in hearing, predominance of prelingual hearing loss was observed in patients with proven genetic deafness. In patients with no identified mutations, the distribution between prelingual and postlingual onset of the hearing impairment was approximately the same. [Figure 6]

Among the selected five families who underwent targeted next-generation sequencing, no probrand was a carrier of mutations in Cx26 (GJB2), Cx30 (GJB6) and Cx31 (GJB3) [Figure 7]. After performing the targeted next-generation sequencing among the selected families, interesting results were received for all who were expected to have gene mutations responsible for hearing. In families: №1, №2 and №4, no currently known mutations were identified in genes associated with hearing. In family №3, through CNV analysis, in the tested patient a duplication of 51 base pairs in the OTOA gene was found (16:21679026-21679076), affecting exon 3 and partially intron 1-2 and intron 2-3. CNVs have been increasingly associated with non-syndromic hearing loss [4].

In family №5 the replacement, p.Gly285Ser, is a mutation leading to loss of functions and has a strong dominant-negative effect on the wild type KCNQ4 channels [5]. It has been proven that in Xenopus oocytes the mutation leads to lack of $\mathrm{K}^{+}$ currents [6]. Dysfunction of KCNQ4 is associ- ated with degenerative processes that have been observed in many other genetic diseases affecting the nervous system. Therefore, hearing loss in patients with mutations in that gene progresses and inheritance in them is autosomal dominant.

\section{Discussion}

Among the Bulgarian population, the most common mutation in gene GJB2 is c. $35 \mathrm{delG}$, found in $43.75 \%$ of the study patients with SNHL: $29.69 \%$ in a homozygous state and in $14.06 \%$ of the cases in a heterozygous. It was identified in patients from Bulgarian and Turkish ethnic origin but not in patients from the Roma ethnic group. Carrier frequency of different mutations in GJB2 differs globally.

According to literature data and in conclusion from our experience with the study group of patients with SNHL, genetic testing must start with search of mutations in the GJB2 gene, as the most common cause of deafness in the Bulgarian population. Therefore screening for mutations among the study participants started with Cx26 gene. Ethnic origin plays an important role in the distribution and frequency of mutations, as it is clear from the obtained results in Ashkenazi Jews (167 delT), Asians (23delC), Caucasians (35delG). These observations were confirmed by our results as well. In the Bulgarian population, among the

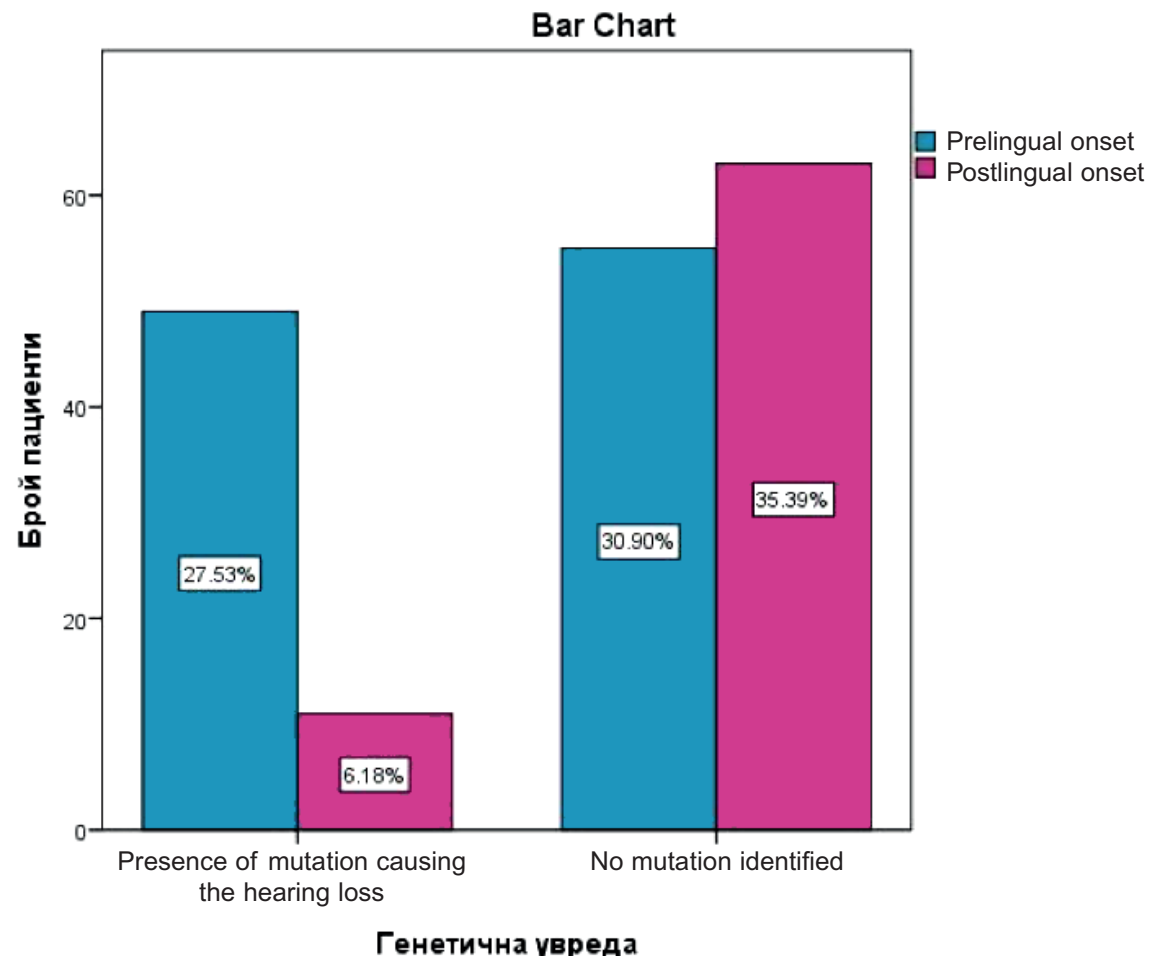

Figure 6. Presence of genetic mutation in connection with the hearing loss onset 


\section{Семейство №1}

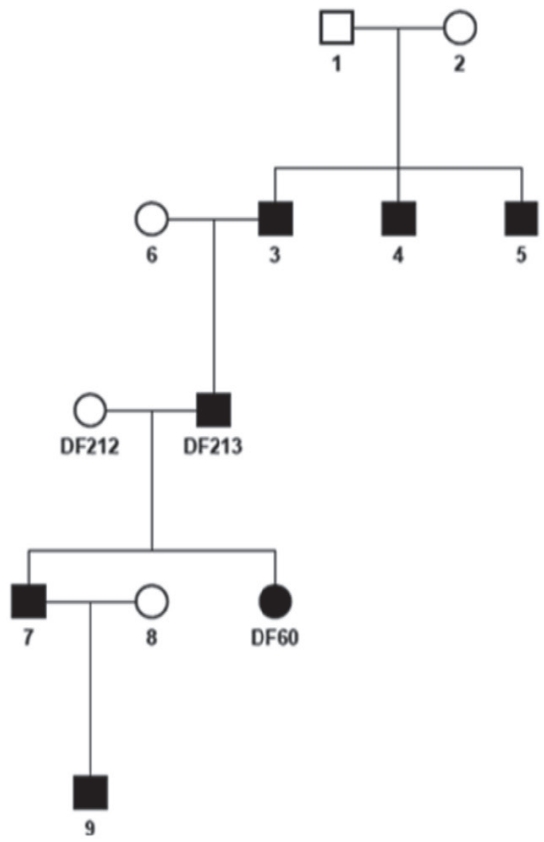

\section{Семейство №2}

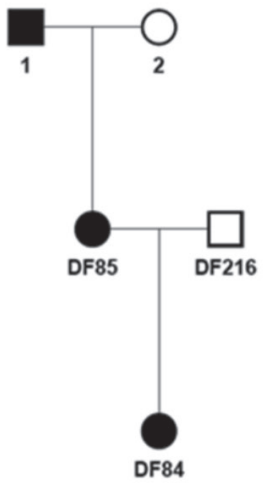

Семейство №3
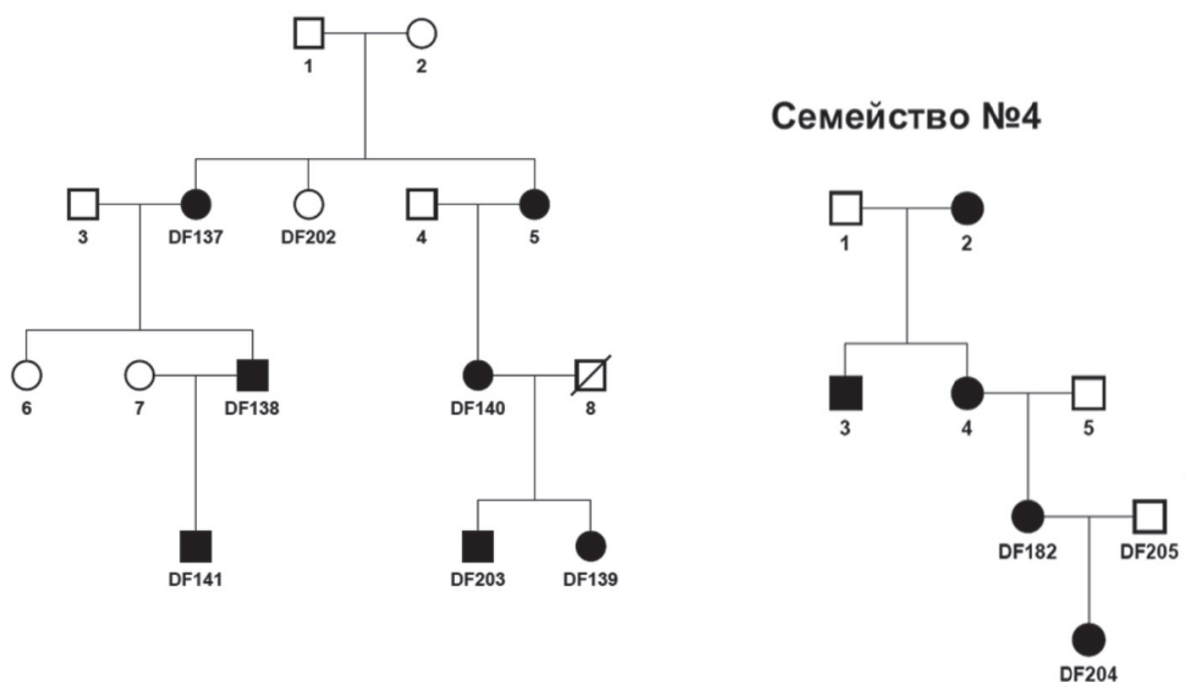

Семейство №5

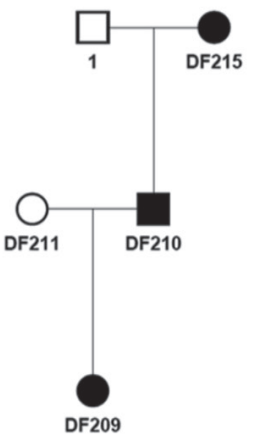

Figure 7. Family trees of the studied families. In family №1 probrand is DF60; in family № 2 probrand is DF84; in family № 3 probrand is DF139; in family № 4 - DF182; in family № 5 - DF210.

tested Roma group with SNHL a high frequency of mutation p.W24X in a homozygous state was observed.

As a next step in diagnosing hereditary hearing loss, following the screening for mutations in connexin genes, we introduced the use of targeted next-generation sequencing in patients in whom such mutation was not found or it was in a heterozygous state.

We selected families where several members of different generations had SNHL and our aim was to identify rare mutations in genes responsible for hearing. After performing the targeted next-generation sequencing among the selected families, interesting results were received for all who were expected to have gene mutations responsible for hearing.

In family №3, in the tested probrand, through CNV analysis, a duplication of 51 base pairs in the OTOA gene (16:21679026-21679076) was found, 
affecting exon 3 and partially intron 1-2 and intron 2-3.

In family №5 the replacement, p.Gly285Ser, is a mutation leading to loss of functions. The hearing loss progresses in patients with mutations in that gene. Inheritance in them is autosomal dominant. Cases of autosomal recessive inheritance have also been reported in carriers of mutations in KCNQ4. Wasano K. et al reported on a family with autosomal recessive form of non-syndromic hearing loss who had mutation c.1044_1051del8 in a homozygous state. The probrand was diagnosed with severe hearing loss that was either congenital or with an onset in early childhood. The probrand's daughter was a heterozygous carrier of the mutation but she had no hearing impairment [Wasano K. et al, 2015].

The found variant in $\mathbf{K C N Q} 4$ co-segregated with the disease in the family and explained the occurrence of the hearing loss. Mutations in that gene have been associated with progressive hearing loss with a postlingual onset. In the probrand's daughter the hearing impairment was diagnosed at the age of 3 months. Currently in literature, there are no described cases of carriers of mutation c. $853 \mathrm{G}>\mathrm{A}$ (p.Gly285Ser), with a congenital hearing impairment. In that girl, the presence of a variant in another gene or the same gene might have aggravated the clinical phenotype.

\section{Conclusion}

Hearing screening in order to identify deafness needs to start in neonatal units within 48 hours after birth. An important step is referring the child to an audiologist in case negative OAEs are registered. The next step is BAEP testing.

Hearing loss is the only sensory deficit, which can be successfully treated even with regard to profound deafness. Cochlear implants have a positive effect in speech production and speech perception. A significant positive change in cognitive ability and reading is achieved, strongly expressed in children with GJB2 mutation, which causes an isolated impairment in the cochlea without impairment of cranial nerve VIII or the CNS.

\section{References}

1. Hereditary Hearing Loss and Deafness Overview, GeneReviews ${ }^{\circledR}$ [Internet].Adam MP, Ardinger HH, Pagon RA, et al., editors.Seattle (WA): University of Washington, Seattle; 1993-2018.

2. Arti Pandya, MD1, Kathleen S. Arnos, PhD2, Xia J. Xia, PhD1, Katherine O. Welch, MS2, Susan H. Blanton, PhD3, Thomas B. Friedman, PhD4 , Guillermina Garcia Sanchez, MD5, Xiu Z. Liu MD, PhD6, Robert Morell, PhD4, and Walter E. Nance, MD, PhD1. Frequency and distribution of GJB2 (connexin 26) and GJB6 (connexin 30) mutations in a large North American repository of deaf probands; Genet Med. 2003 Jul-Aug;5(4):295-303.

3. Nishio SY, Usami S. Deafness gene variations in a 1120 non-syndromic hearing loss cohort: Molecular epidemiology and deafness mutation spectrum of patients in Japan. Ann Otol Rhinol Laryngol 2015;124 (Suppl 1):49S-60S.

4. Sloan-Heggen CM. et al, 2016; Shearer AE. Et al, 2014

5. Mencía A. Et al. 2008; Gao Y. et al, 2013

6 Kubisch C. et al, 1999. 